\title{
A Time Series Analysis of the Relationship Between Ambulatory EMG, Pain, and Stress in Chronic Low Back Pain
}

\author{
Michael E. Geisser \\ Department of Physical Medicine and Rehabilitation, University of Michigan \\ Michael E. Robinson ${ }^{1}$ and Charles Richardson \\ Department of Clinical and Health Psychology, University of Florida
}

\begin{abstract}
Twenty-one subjects with chronic back pain (CBP) participated in an ambulatory electromyography (EMG) monitoring study to ascertain the relationships between muscle activity, physical activity, psychosocial stress, and pain. A time-series analysis approach was adopted to investigate both immediate and lagged associations between these variables in an attempt to determine potential causal relationships. Results for group relationships showed a significant relationship between physical activity and pain, self-report of stress and pain, but no relationship between EMG activity and pain. A lagged relationship between physical activity and pain was found, suggesting a causal relationship between physical activity and pain. However, no time lag was observed between stress and pain, hence no causal relationship can be elucidated. Analysis at the individual level indicated stronger relationships between several combinations of these variables, highlighting the need to consider the heterogeneity of the CBP population and etiology of CBP. The use of ambulatory monitoring of pain, stress, and EMG is suggested as one avenue to further explore the population's heterogeneity.
\end{abstract}

Descriptor Key Words: ambulatory EMG; self-monitoring; back pain; stress; time-series analysis.

${ }^{1}$ Address all correspondence to Michael E. Robinson, Ph.D., Box 100165 HSC, Department of Clinical and Health Psychology, University of Florida, Gainesville, Florida 32610. 
Musculoskeletal disorders have become a major focus of concern for both employers and health care professionals. Chronic back pain (CBP) has been a particularly troublesome variant of the musculoskeletal disorders. Health researchers remain divided as to the origin of CBP. This is due in part to the lack of a comprehensive model that can explain the seemingly discordant results found throughout the CBP literature. Within this collection of research, there are two major etiological models of CBP: the biomechanical model and the reflex-spasm model (Dolce \& Raczynski, 1985). The biomechanical model proposes that $\mathrm{CBP}$ may be a result of abnormally low levels of muscle activity during movement or right-left electromyographic (EMG) asymmetries. This theory suggests that abnormal EMG activity patterns are a result of poor posture and guarding which develop in response to the original insult (Cram \& Steger, 1983). It is believed that such irregular muscle activity provides abnormal support for the spine, which in turn becomes unstable. The instability of the spine enhances the possibility of infringement upon nerve endings and thereby produces pain (Ahern, Follick, Council, Laser-Wolston, \& Litchman, 1988; Dolce \& Raczynski, 1985; Wolf, Nacht, \& Kelly, 1982). In the reflex-spasm model, there are two competing hypotheses explaining the etiological factors that produce CBP. One of the two emphasizes the importance of physical stressors, while the other proposes that psychophysiological factors underlie the development of CBP. Regardless of whether the initial damage is caused by physical or psychophysiological factors, both hypotheses agree that eventually a reflex-spasm develops (Cobb, deVries, Urban, Luekens, \& Bagg, 1975; Dolce \& Raczynski, 1985; Miller, 1985; Nouwen \& Bush, 1984).

In support of the biomechanical model, patients with chronic low back pain (CLBP) have been found to display lower EMG levels compared to controls during movement (Ahern et al., 1988; Collins, Cohen, Naliboff, \& Schandler, 1982; Wolf \& Basmajian, 1978; Wolf, Basmajian, Russe, \& Kutner, 1979; Wolf et al., 1982). In addition, Cram and Steger (1983) reported greater EMG asymmetries for CBP patients compared to controls. Hoyt et al. (1981) found that CBP patients displayed greater asymmetries while sitting, but not standing. In contrast, Ahern et al. (1988) did not find significant EMG asymmetries for CBP subjects during dynamic movements. Other studies contradict the predictions of the biomechanical model and provide evidence in support of the reflex-spasm model.

Similar to the biomechanical model, studies testing the reflex-spasm model have produced mixed results. Some researchers have found that increased muscle activity is associated with greater pain. Cobb et al. (1975) demonstrated that EMG activity level increases in response to painful stimulation. Jayasinghe, Harding, Anderson, and Sweetman (1978) and Hoyt et al. (1981) found greater muscle activity for CBP patients compared 
to controls during standing, although Hoyt et al. (1981) found no differences in muscle activity between the two groups while in the supine, prone, or sitting positions. In addition, Kravitz, Moore, and Glaros (1981) found that although resting EMG activity did not differ between CBP patients and controls, the EMG activity of the two groups was different during cocontraction relaxation. DeGood, Stewart, Adams, and Dale (1989) reported that CBP patients have greater resting EMG levels, greater EMG reactivity to emotional stressors, and greater left-right asymmetries. Finally, Nigl and Fischer-Williams (1980) trained four back pain patients in EMG biofeedback and relaxation, and as predicted by the reflex-spasm theory, all four subjects displayed significantly lower EMG activity and experienced substantial pain relief following treatment.

Other studies have not supported the reflex-spasm model. For example, Miller (1985) found no difference between CBP patients and controls in their EMG muscle activity during standing, quiet sitting, and active sitting. Likewise, Cohen, Swanson, Naliboff, Schandler, and McArthur (1986) measured heart rate, skin conductance, and EMG at six muscle sites during cold pressor tasks, mental arithmetic, and while assuming various postures. They found no significant differences between 13 CBP patients and 13 controls in any of these conditions.

Some researchers have proposed other models to explain these discrepancies. One possible explanation is that many chronic back pain patients suffer from various disorders with differing etiological causes and are artificially lumped into one group (Arena, Sherman, Bruno, \& Young, 1989). Most studies include subjects with CBP regardless of the type of pain or pathophysiology involved. To test this, the authors divided subjects into five groups based on their diagnostic type of back pain. In general, EMG activity levels varied as a function of movement and diagnostic subgroup. However, the group differences obtained were small. Other factors that may contribute to the discrepant findings in the literature include recording EMG activity in only one or two body positions (Sherman, 1985), small sample sizes, failure to report the pain level of subjects during the experiment (Sherman, 1985), and static rather than dynamic assessment of EMG. Even the studies that measure EMG activity during dynamic movement do so for only a short period of time, usually in a laboratory setting.

To examine more naturalistic and continuous muscle activity, Feuerstein (1986) designed a study in which he monitored the EMG muscle activity of CBP patients and normal controls during eight hours of regular activity over a three-day period. He compared EMG levels, pain and mood ratings, and autonomic arousal. His results suggested that the paraspinal muscle activity of CBP patients did not differ from controls nor was it cor- 
related with pain ratings. However, some limitations of this study include limited knowledge of the subjects' diagnoses, and the fact that the CBP subjects may have been high functioning and not representative of the CBP population in general as the CBP subjects did not significantly differ from controls in their daily activity ratings. In addition, the statistical analyses did not allow for examination of delayed relationships between muscle activity and pain, as some authors have proposed that increases in pain and muscle tension may not occur simultaneously.

Sherman (1988) suggested that both the use of an ambulatory EMG monitor and a time series statistical analysis would likely lead to clearer results. Indeed, in his presentation of four case studies in which he followed these procedures, Sherman found that perceived pain and muscle activity levels for CBP patients are correlated following a delay period, although the physiological mechanisms that might explain this are unclear.

In the present study, we used ambulatory monitors to measure EMG activity among chronic pain patients while they engaged in their normal daily activities over an extended period of time. Patients also provided ratings of stress, positive and negative mood, activity, and pain every $30 \mathrm{~min}$ utes. A time series analysis was used to examine relationships between an individual's ratings and muscle activity. By utilizing these techniques, we attempted to examine a crucial assumption of the reflex-spasm theory: whether changes in muscle. activity are correlated with changes in experienced pain levels.

The first hypothesis (EMG activity-pain) of this study is that EMG activity and pain intensity are correlated such that increases in pain intensity are related to increases in muscle activity. Secondly, because higher levels of physical activity are generally associated with increased muscle activity, it was predicted that physical activity would be correlated with experienced pain (physical activity-pain hypothesis). The third hypothesis is that the association between muscle activity and pain should be stronger following a delay period. In addition, the question of whether psychophysiological factors as proposed by the reflex-spasm theory are relevant to the maintenance of CBP was also explored. This model predicts that EMG activity and experienced pain of CBP patients should correlate with psychosocial stress. Thus, the fourth (psychosocial-pain) and fifth (mediation) hypotheses propose that there is a relationship between psychosocial variables and pain, and it is mediated by muscle activity. Although a clear causal link cannot be established with this design, the claims of such a causal relationship will be enhanced if a temporal relationship is established between the psychosocial variables as well as the other predictor variables and pain intensity. Therefore, the sixth (directionality) hypothesis is that changes in the predictor variables should precede changes in pain, and that 
this relationship should be stronger than any competing association in which changes in pain intensity precede changes in the "predictor" variables.

\section{METHOD}

\section{Participants}

Twenty-five subjects between the ages of 21 and $59(M=35.1)$ who were diagnosed with CBP volunteered to participate in this study. All but one subject experienced low back pain. Eleven subjects were female and fourteen were male. All were Caucasian. Two female and one male subject dropped out of the study after one day. A fourth male subject was excluded from the analysis because of a lack of proper participation. Six of the 21 remaining subjects were obtained from an outpatient multidisciplinary spinal rehabilitation program while the other 15 were participants of an outpatient physical therapy group. Each had endured CBP for at least 6 months with a range between 7 and 108 months and an average of 34 months. All 21 subjects were ambulatory. In addition, all but one of the outpatient physical therapy groups were currently employed or attending school. Each of the spine program participants were receiving disability through Workers Compensation. A single diagnosis was obtained for 18 of the 21 final subjects. The diagnosis of each subject varied substantially, and a list of the primary diagnoses included degenerative disk disease (4 subjects), myofascial pain (3), spondylolisthesis (2), cervical myeloradiculopathy, postlaminectomy pain syndrome, congenital fusion, thoracic spinal disease, lumbar radicular pain, sciatica, scoliosis, lyticpars defect, and vertebral fracture. Three subjects had multiple diagnoses.

\section{Procedure}

After agreeing to participate, each subject was given a demonstration of how to place the EMG monitor system on their body. Subjects were instructed to secure an elastic belt around their waist such that the BioFlex $^{\mathrm{TM}}$ (Physical Health Devices) silver imbedded cloth electrodes (1 $\times 5$ in.) were placed bilaterally above the paraspinal muscle approximately $5 \mathrm{~cm}$ from the L3 disk. The experimenter placed the electrodes initially, and the subject was instructed to leave them in the belt. Subjects had only to put on the belt during the recording period. The belt placement was 
marked in ink on the skin. The reference electrode ran parallel to the right active electrode with a separation of approximately 1 in. The electrodes were attached by Velcro to the experimental belt. The EMG monitor itself was contained in a pouch, which was attached to the subjects' regular pants belt. Subjects turned on the machine during the recording period.

The Bio-Prompt 3000 patient module (Empi, Inc., St. Paul, Minnesota) was used for ambulatory EMG recording. The raw EMG signal is amplified, filtered, converted from an ac to dc voltage proportional to the RMS voltage, and finally digitally sampled. The analog signal processing flow of the device is as follows: autogain amplification, bandpass filtering of 100 to $540 \mathrm{~Hz}$, notch filtering at $60 \mathrm{~Hz}$, true RMS to dc conversion, and a digital sampling rate of $128 \mathrm{~Hz}$. The EMG module is battery powered with dimensions, in inches, of $1.5 \mathrm{~h} \times 3.5 \mathrm{w} \times 6 \mathrm{l}$ and weighs $16 \mathrm{oz}$. Since the device is a single-channel recorder, the signals from the right and left paraspinal musculature were averaged and then recorded for a gross measure of muscle activity from the low back area. An average EMG activity level was recorded by the EMG monitor for every one and one half minutes. These data points were averaged to obtain an EMG activity score for every half hour of the recording period. The EMG monitor was worn for three days. Subjects were instructed to wear the recorder for 10 continuous hours each of the three days, although actual wear time averaged 8.1 hours (range of 3.5 to $10.5 \mathrm{~h}$ ). Aside from wearing the EMG monitor, subjects also estimated their current pain level on a $8-\mathrm{cm}$-long anchored visual analogue scale (VAS). In addition, they recorded their current level of activity, mood, and psychosocial stress. The VAS were anchored in the following manner: Pain, no pain to worst imaginable pain; Stress, no stress to worst imaginable stress; positive mood (happy or serene), not at all positive to extremely positive; negative mood scale (angry, sad, anxious), not at all negative to extremely negative. The VAS were kept in a pocket-sized notebook, which also contained the instructions for placing the EMG monitor system on the subject's body. VAS scales are widely used to assess pain (Huskisson, 1983). In addition, previous studies have suggested that VAS scales of mood are simple and valid methods of measuring subjective feelings (Sift, 1989), particularly global measures of mood (Morrison \& Peck, 1990). Subjects were also given a watch with an alarm that sounded every half hour as a reminder to complete the next set of VAS. They were encouraged to maintain their normal daily routine. To assure temporal alignment of EMG recordings and ratings, subjects were instructed to simultaneously turn on the EMG recorders and start the watch alarms. 


\section{Data Analysis}

As previously mentioned, an average EMG activity level was recorded by the EMG monitor for every 90 -s period. Once these 90 -s averages were tabulated, they were inspected for any unlikely readings (any rating below .1 or above 125). All unlikely readings were discarded such that they did not enter into the calculations for a 30-min average EMG activity score. As a result, a small number of 30-min average EMG activity scores either were based on minimal number of data points or were unable to be estimated because of a lack of available EMG data. As a consequence, missing EMG activity scores were estimated by taking the average of the nearest available EMG activity scores preceding and following the missing EMG activity score. Mean EMG levels for all subjects across all 3 days were averaged $($ mean $=9.04 \mu \mathrm{V}, \mathrm{SD}=7.0)$.

To test the major hypotheses of this study, a time series regression procedure was used to determine the relationship between pain and the predictor variables (positive and negative mood, stress, physical activity, and EMG activity). As previously noted, autocorrelation is often a problem for standard regression analysis. In the analysis of time-series data, spurious relationships can come about because the data are serially dependent, that is, the value of a variable is in part dependent on its preceding value(s). The degree of serial dependence, or the correlation between a time series with the same series, lagged, is termed autocorrelation. To remove serial dependence in the data, time series regressions were conducted, which corrected for autocorrelation using the exact maximum likelihood method. With pain as the dependent measure, autocorrelation was found in $38 \%$ of the daily ratings using the Box-Ljung statistic.

This time series analysis was calculated for each individual daily record for each individual subject. Thus, a total of 63 time series analyses were conducted. To determine whether there was a concurrent relationship between pain and the predictor variables across subjects, all 63 beta scores for each predictor variable were averaged to determine a mean beta score for each predictor variable. Because one subject for one day did not wear the EMG monitor for a sufficient period of time, only 62 beta scores were averaged for the EMG activity variable. Likewise, owing to either insufficient response variability or nonquantifiable responses, one day was not analyzed for both negative mood and physical activity, and thus, only 62 beta scores were available for averaging with these predictor variables. To examine whether the beta weights were significantly different from zero across all subjects, multiple regression analyses were conducted, with subjects and day as independent variables. These variables were effect coded, 
and a $t$-test was computed to determine if the intercept was significantly different from 0 .

\section{RESULTS}

Descriptive statistical analysis revealed that the mean rating scores for each variable across subjects were generally low. On a 0 - to $8-\mathrm{cm}$ scale, the average ratings were: pain $(M=2.04, S D=1.95)$, stress $(M=1.76$, $S D=1.63)$, positive $\operatorname{mood}(M=3.73, S D=2.08)$, negative $\operatorname{mood}(M=$ $1.42, S D=1.49)$ and physical activity $(M=2.30, S D=1.78)$. Results indicated that the average beta score for stress $(M=.18, S D=.53)$ with pain as the dependent variable was significantly different from zero $(t=$ $2.91, p=.006)$, suggesting a positive relationship across subjects. In addition, the relationship between physical activity $(M=.24, S D=.42)$ and pain was also found to be significant and positive $(t=4.48, p<.01)$. No other significant relationships or trends were found. Neither the subjects nor days effects were significant (see Table I).

Because Sherman (1988) found a strong EMG-pain relationship after a delay period, a series of cross correlation lag procedures ranging from a lag of three hours to a lag of 30-min were conducted to determine whether a relationship between the predictor variables and pain might exist. The strongest relationships occurred concurrently and at a 30-min delay. As a result, the time series procedure described above was repeated such that the relationship between the predictor variables and pain ratings at a lag

Table I. GROUP EFFECT-BETA WEIGHTS Dependent Variable: PAIN

\begin{tabular}{lccccc}
\hline Independent variable & Mean & $S D$ & $N$ & $t$-score & $p$-value \\
\hline \multicolumn{5}{c}{ No Delay } \\
Stress & .18 & .53 & 63 & 2.91 & .01 \\
Positive mood & -.03 & .47 & 63 & -.50 & $N S$ \\
Negative mood & .09 & .46 & 62 & 1.77 & $N S$ \\
Activity & .24 & .42 & 62 & 4.48 & .01 \\
EMG & -.02 & .32 & 62 & -.48 & $N S$ \\
& Delayed pain (30 min) & & \\
Stress & .07 & .70 & 62 & .94 & $N S$ \\
Positive mood & -.13 & .75 & 61 & -1.29 & $N S$ \\
Negative mood & -.07 & 1.11 & 61 & -0.32 & $N S$ \\
Activity & .17 & .48 & 61 & 2.34 & .01 \\
EMG & .02 & .46 & 62 & .37 & $N S$ \\
\hline
\end{tabular}


of 30-min was examined. Results from the $t$-tests for the mean beta scores suggested that only physical activity $(M=.17, S D=.48)$ was significantly different from zero. No subject or days effect was observed (see Table I).

In addition, because Sherman (1988) had found that the peak association between EMG activity and pain occurred at a three hour delay, a similar time series procedure was repeated with a three hour lag and the mean beta score for EMG activity was calculated. No significant relationship was found. Several studies researching the relationship of stress on health have found either small effects or no effect at all when examining across subjects. However, the authors of these studies have noted that upon examination of the results for individual subjects, a significant minority display a strong relationship between stress and symptomatology (Kohler \& Haimerl, 1990; Hazlett \& Haynes, 1992). Therefore, each individual time series analysis for each subject was examined to determine whether any of the predictor variables were associated with pain concurrently or at a 30min delay. Because each subject was tested for three days, it was decided that a predictor variable must at least show a trend toward significance ( $p$ $<.10$ ) for at least two of the three test days for that variable to be considered as having a meaningful association with a given subject's pain experience. The advantage of a two-day minimum requirement is that it significantly reduces the likelihood of chance findings. Based upon the individual analysis, a relationship between at least one predictor variable and concurrent pain was found for 9 of 21 subjects (see Table II). Stress was positively associated with concurrent pain for five subjects, while four subjects displayed a positive relationship between physical activity and pain. In addition, positive mood was positively correlated with pain for two subjects and negatively correlated for a third. Negative mood was positively associated with pain for only one subject.

The relationship between lagged pain and at least one predictor variable was meaningful for 6 of the 21 subjects (see Table II). Given that three of these subjects had not displayed any significant relationship between the predictor variables and concurrent pain, the total number of subjects displaying a meaningful relationship between one predictor variable and pain (concurrent and lagged) was 12 of 21 . Physical activity was the most commonly associated variable with lagged pain (three subjects) along with EMG activity which was positively correlated for two subjects and negatively associated for a third. Stress was positively connected with lagged pain for one subject and negative mood was negatively correlated for another. Again, the subject and days effects were not significant.

The mediation hypothesis predicted that the effect of psychosocial variables on pain was mediated by EMG activity. To address this question, the time series regression procedure which was employed with concurrent 
Table II. INDIVIDUAL ANALYSIS Dependent Variable:

PAIN

\begin{tabular}{ll}
\hline Subject & \multicolumn{1}{c}{\begin{tabular}{c}
\multicolumn{1}{c}{ Independent variable } \\
(days significant)
\end{tabular}} \\
\hline & \multicolumn{1}{c}{ No delay } \\
3 & Stress (2), activity (2) \\
5 & Stress (2) \\
6 & Stress (2) \\
8 & Positive mood (2) \\
11 & Negative mood (2) \\
15 & Stress (2), positive mood (2), activity (2) \\
16 & Stress (2), positive mood (2) \\
17 & Activity (2) \\
25 & Activity (2) \\
& \multicolumn{1}{c}{ Delayed pain (30 min) } \\
& Stress (2) \\
6 & Negative mood (3), EMG (2) \\
7 & Activity (2) \\
9 & EMG (2), \\
11 & EMG (2), activity (2) \\
16 & Activity (2) \\
23 &
\end{tabular}

and delayed pain as the dependent measures was repeated using EMG activity (concurrent and lagged) as the dependent measure. Results across subjects indicated that physical activity was the only predictor variable correlated with EMG activity. In the concurrent EMG activity analysis, the mean beta score for physical activity $(M=.20, S D=.48)$ was positively associated with EMG activity $(t=3.61, p<.01)$, while in the lagged EMG activity analysis, the mean beta score for physical activity $(M=.13, S D=$ $.55)$ and EMG activity approached significance $(t=1.96, p<.1)$. There were no other significant relationships or trends (see Table III). The subjects and days effects were not significant.

As had been employed with pain as the dependent variable, an analysis of each individual subject was conducted to determine whether the predictor variables were associated with EMG activity for a significant portion of the subjects. The same criterion for establishing a meaningful relationship between the predictor variables and an individual's pain was utilized in this analysis of EMG activity (two days of at least a trend toward significance for a given predictor variable). Results indicated that a significant relationship between at least one of the predictor variables and concurrent EMG activity was found for only two subjects. In both cases, physical ac- 
Table III. GROUP EFFECTS-BETA WEIGHTS Dependent Variable:

EMG

\begin{tabular}{lccccc}
\hline \multicolumn{1}{c}{$\begin{array}{c}\text { Independent } \\
\text { variable }\end{array}$} & Mean & $S D$ & $N$ & $t$-score & $p$-value \\
\hline & \multicolumn{5}{c}{ No delay } \\
Stress & .09 & .54 & 62 & 1.28 & $N S$ \\
Positive mood & .04 & .48 & 62 & 0.44 & $N S$ \\
Negative mood & .10 & 1.13 & 61 & 0.59 & $N S$ \\
Activity & .20 & .48 & 61 & 3.61 & .01 \\
& \multicolumn{7}{c}{ Delayed (30 min) } & & \\
Stress & .11 & .56 & 61 & 1.60 & $N S$ \\
Positive mood & .10 & .98 & 61 & 0.71 & $N S$ \\
Negative mood & .00 & .86 & 60 & 0.00 & $N S$ \\
Activity & .13 & .55 & 60 & 1.96 & .10 \\
\hline
\end{tabular}

Table IV. INDIVIDUAL ANALYSIS Dependent Variable: EMG

\begin{tabular}{ll}
\hline Subject & \multicolumn{1}{c}{ Independent variable (days significant) } \\
\hline & \multicolumn{1}{c}{ No delay } \\
19 & Activity (2) \\
23 & Activity (2) \\
& Delayed EMG (30 min) \\
1 & Activity (2), stress (2) \\
2 & Activity (2) \\
8 & Activity (2), positive mood (2) \\
15 & Activity (2) \\
\hline
\end{tabular}

tivity was the predictor variable positively associated with EMG activity. Similarly, the analysis of the relationship between the predictor variables and 30-min delayed EMG activity revealed that four additional subjects displayed a positive association between physical activity and EMG activity. Furthermore, a positive relationship between stress and EMG activity was also found for one of these four subjects. Likewise, a positive association between positive mood and lagged EMG activity was found for another (see Table IV).

In relationship to the directionality hypothesis, it has been commonly suggested that CBP patients' stress, mood, and activity levels are very sensitive to their experience of pain, and actually are more likely to follow 
Table V. Group Effects-Beta Weights PAIN as PREDICTOR (30 min)

\begin{tabular}{lcccrc}
\hline \multicolumn{1}{c}{$\begin{array}{c}\text { Independent } \\
\text { variable }\end{array}$} & Mean & $S D$ & $N$ & $t$-score & $p$-value \\
\hline Stress & .08 & .56 & 62 & .97 & $N S$ \\
Positive mood & .09 & .48 & 62 & 1.39 & $N S$ \\
Negative mood & -.07 & .56 & 61 & -0.93 & $N S$ \\
Activity & .05 & .40 & 61 & 1.24 & $N S$ \\
EMG & .05 & .35 & 62 & 1.08 & $N S$ \\
\hline
\end{tabular}

changes in pain, rather than precede it (Nouwen \& Bush, 1984). As a result, a series of time series analyses was repeated with the "predictor" variables being lagged by 30 -min such that pain ratings preceded the "predictor" variables by $30-\mathrm{min}$. Mean beta scores were then calculated for each "predictor" variable. Results indicated that there were no significant relationships between pain and any of the lagged "predictor" variables (see Table $\mathrm{V}$ ). In addition, individual analyses were performed in the manner previously described (meaningful relationship criterion for individual subject: two days of at least a trend toward significance for a given predictor variable) and only two subjects displayed an association between pain and a lagged "predictor" variable. Lagged physical activity was negatively correlated with pain for one subject and lagged negative affect was negatively associated with pain for another.

\section{DISCUSSION}

Results of this study indicated mixed support for the proposed hypotheses. First, in opposition to the expectations of the mediation, delay, and EMG activity-pain hypotheses, there were no significant relationships between EMG activity and pain intensity. In support of the physical activity-pain hypothesis, however, a significant correlation was found between physical activity and pain intensity. Furthermore, because of the temporal nature of the association, it is likely that physical activity is causally related to pain intensity. Another hypothesis (psychosocial pain) was also supported, suggesting that stress and pain intensity are correlated. Unlike the relationship between physical activity and pain, no delayed effects were significant between stress and pain, therefore no support was found for the directionality hypothesis. Although stress and physical activity were associated with pain intensity, the effect size was actually small. Case by case analysis suggested a stronger relationship between these variables for a sub- 
set of the population. The possibility that a causal relationship exists between pain and physical activity is enhanced by the fact that a small correlation was found for perceived physical activity that precedes pain by 30 min. Certainly, a true experimental design would be needed to ferret out a possible spurious relationship. Nonetheless, the fact that a temporal relationship exists enhances the likelihood of a causal link. In addition, a positive relationship between physical activity and pain intensity is consistent with CBP patients' self-report and other research findings (Matheson, Macintyre, Taunton, Clemont, \& Lloyd-Smith, 1989).

Despite finding significant relationships between physical activity and pain, the effect size was in the small range for psychosocial research studies (Rosnow \& Rosenthal, 1989). The small size of this relationship is not surprising given the multitude of competing variables that may play a role in perceived pain intensity (stress, mood, diet, weather, body position, and hormonal and other biochemical changes). In addition, perceived level of physical activity is a gross, subjective measure of actual physical activity and a significant portion of actual activity may have been inaccurately assessed. Thus, the relationship may have been stronger if more objective measures had been employed. Furthermore, the person's perceived activity level was a general measure of physical activity rather than a specific assessment of activity of the lumbar muscles. Thus, the degree of correspondence between the two may have been small. For example, sitting in certain positions can be strenuous for the lumbar muscles, yet a subject is likely to rate the activity as low. Given enough of these dissociations, the correlation between EMG activity in the lumbar region and physical activity ratings would likely be small. The fact that the physical activity-pain hypothesis was supported did little to enhance any of the major CBP theories. Because the spasm-reflex and biomechanical models of CBP all predict that increased physical activity should be associated with increased pain intensity, the results supporting the physical activity-pain hypothesis are consistent with each of these theories. In the individual analyses, data from one subject supported the biomechanical model (EMG was inversely related to lagged pain on two days), and the reflex spasm model was supported in two subjects. These results suggest, as noted further below, that patients with chronic pain are heterogeneous in terms of their make-up and factors that are related to the perpetuation of their pain. Inconsistencies noted in previous studies examining the relationship between EMG activity and pain may be due to sample differences. Ultimately, it may be of interest to examine what factors separate patients who develop a muscle tension or fatigue contribution to their chronic pain from those who do not.

Self-report of level of stress was the only predictor variable besides physical activity to be significantly correlated with pain intensity. In addi- 
tion, because increased stress is associated with increased pain intensity, it is consistent with the psychophysiological variant of the spasm-reflex model. Unfortunately, the direction of the relationship is not clear, as no lagged relationships were observed. The degree of association, on the other hand, was not very large. In addition, there appeared to be a great deal of variation between stress, mood and pain across subjects. This may be related to differences in the criteria to assess mood and stress or individual differences in persons' awareness of their bodily states and mood. Furthermore, the relationships between pain and mood were inconsistent. The relationship between pain and mood was positive for two subjects and negative for one subject. While the latter relationship is supported by studies suggesting that dysphoric mood states are an understandable consequence of unrelenting pain (Gamsa, 1990), the positive relationships between positive mood and pain may represent coping attempts to maintain "a positive attitude" in response to pain flare-ups. Lastly, mood and stressors may be related to different aspects of the pain experience. According to multidimensional theories of the pain experience (Melzack \& Casey, 1968; Turk \& Rudy, 1986), the impact of these variables may vary according to which component of pain is examined.

From a clinical perspective, the results suggest that the relationship between pain, activity, mood, and stress needs to be evaluated on a caseby-case basis. For some individuals, managing their mood and stressors may have a significant impact on their experience of pain while other patients may benefit more from activity pacing therapies.

One potential shortcoming of the present study is that ratings were not taken frequently enough to observe relationships that may be rapid and changing. For example, Sherman (1988) found evidence to suggest a rapidly changing association between muscle activity and perceived pain. Furthermore, it was assumed that the relationship between pain and muscle activity was primarily unidirectional for a given individual. It is quite possible that direction of the relationships may vary over time within a given individual. A number of subjects failed to wear the ambulatory EMG device or had bad/missing data in their records. Though there was no apparent systematic bias to these events, such biases could have influenced relationships among measures. Closely monitored use of these devices may enhance data integrity and yield more clear relationships among variables.

Similar findings to the ones obtained in the current study have been found with headache patients. Hatch, Prihoda, Moore, Cyr-Provost, Borcherding, Boutros, and Seleshi (1991) measured frontalis EMG activity and employed a time series design to assess whether EMG activity was correlated with headache intensity. Results suggested that EMG activity was not related to changes in pain intensity across subjects, although one-third of 
the subjects (4/12) displayed an association. The direction of the relationship, however, varied for this subset such that two of the four subjects displayed a positive association and the other two a negative association. The EMG activity of headache patients was not found to differ from controls, even during low-activity, high-pain periods (e.g., sleeping with a headache). As with this study, there was also a significant relationship between concurrent stress and pain, but no delayed effects in either direction.

Results of this study highlight the heterogeneity of patients and contributing factors in the chronic low back pain population. Ambulatory monitoring of EMG, anxiety, mood, and pain are likely to yield different results for different individuals. Future research using the type of technology in this study may need to use more frequent averaging or measurement of dependent variables to better assess the temporal relationships among variables thought to be contributory to pain and function. In addition, given the limitations inherent in a relatively unstructured activity recording task, it may be beneficial to have a more formalized ambulatory activity regimen to assess the relationship between muscle activity and other contributory variables.

\section{REFERENCES}

Ahern, D. K., Follick, M. J., Council, J. R., \& Laser-Wolston, N. (1988). Comparison of lumbar paravertebral EMG patterns in chronic low back pain patients and nonpatient controls. Pain, 34, 153-160.

Arena, J. G., Sherman, R. A., Bruno, G. M., \& Young, T. R. (1989). Electromyographic recordings of 5 types of low back pain subjects and nonpatient controls in different positions. Pain, 37, 57-65.

Cobb, C. R., deVries, H. A., Urban, R. T., Luekens, C. A., \& Bagg, R. J. (1975). Electrical activity in muscle pain. American Journal of Physical Medicine, 54, 80-87.

Cohen, M. J., Swanson, G. A., Naliboff, B. D., Schandler, S. L., \& McArthur, D. L. (1986). Comparison of electromyographic response patterns during posture and stress tasks in chronic low back pain patterns and control. Journal of Psychosomatic Research, 30, 135-141.

Collins, G. A., Cohen, M. J., Naliboff, B. D., \& Schandler, S. L. (1982). Comparative analysis of paraspinal and frontalis EMG, heart rate, skin conductance in chronic low back pain patients and normals to various postures and stress. Scandinavian Journal of Rehabilitation, $14,39-46$.

Cram, J. R., \& Steger, J. C. (1983). EMG scanning in the diagnosis of chronic pain. Biofeedback and Self-Regulation, 8, 229-241.

DeGood, D., Stewart, W., Adams, L., \& Dale, J. A. (1989, March). Paraspinal EMG reactivity of back pain patients and controls. Paper presented at the 10th Annual Meeting of the Society for Behavioral Medicine, San Francisco, CA.

Dolce, J. J., \& Raczynski, J. M. (1985). Neuromuscular activity and electromyography in painful backs: Psychological and biomechanical models in assessment and treatment. Psychological Bulletin, 97, 502-520. 
Feuerstein, M. (1986, March). Ambulatory monitoring of paraspinal skeletal muscle, autonomic and mood-pain interaction in chronic low back pain. Paper presented at the 7th Annual Meeting of the Society for Behavioral Medicine, San Francisco, CA.

Flor, H., Turk, D. C., \& Birmbaumer, N. (1985). Assessment of stress-related psychophysiological reactions in chronic back pain patients. Joumal of Consulting and Clinical Psychology, 53, 354-364.

Gamsa, A. (1990). Is emotional disturbance a precipitator or consequence of chronic pain? Pain, 42, 183-195.

Gift, A. (1989). Visual analogue scales: Measurement of subjective phenomenon. Nursing Research, 38, 286-288.

Hatch, J. P., Prihoda, T. J., Moore, P. J., Cyr-Provost, M., Borcherding, S., Boutros, N. N., \& Seleshi, E. (1991). A naturalistic study of the relationships among electromyographic activity, psychological stress, and pain in ambulatory tension-type headache patients and headache free controls. Psychosomatic Medicine, 53, 576-584.

Hazlett, R. L., \& Haynes, S. N. (1992). Fibromyalgia: A time series analysis of the stressor-physical symptom association. Journal of Behavioral Medicine, 15, 541-557.

Hoyt, W. H., Hunt, H. H., Depauw, M. A., Bard, D., Shaffer, F., Passias, J. N., Robbins, D. H., Runyon, D. G., Semrad, S. E., Symonds, J. T., \& Watt, K. C. (1981). Electromyographic assessment of chronic low-back pain syndrome. Journal of the American Osteopathic Association, 80, 728-730.

Huskison, E. C. (1983). Visual analogue scales. In R. Melzack (Ed.), Pain measurement and assessment (pp. 33-37). New York: Raven Press.

Jayasinghe, W. J., Harding, R. H., Anderson, J. A. D., \& Sweetman, B. J. (1978). An electromyographic investigation of postural fatigue in low back pain - A preliminary study. Electromyography and Clinical Neurophysiology, 18, 191-198.

Kohler, T., \& Haimerl, C. (1990). Daily stress as a trigger of migraine attacks: Results of thirteen single-subject studies. Journal of Consulting and Clinical Psychology, 5, 887-872.

Kravitz, E., Moore, M. E., \& Glaros, A. (1981). Paralumbar muscle activity in chronic low back pain. Archives of Physical Medicine and Rehabilitation, 62, 172-175.

Matheson, G. O., Macintyre, J. G., Taunton, J. E., Clement, D. B., \& Lloyd-Smith, R. (1989). Musculoskeletal injuries associated with physical activity in older adults. Medicine and Science in Sports and Exercise, 21, 379-385.

Melzack, R., \& Casey, K. L. (1968). Sensory, motivational and central control determinants of pain: A new conceptual model. In D. Kenshalo (Ed.), The skin senses (pp. 168-194). Springfield, IL: Charles C. Thomas.

Miller, D. J. (1985). Comparison of electromyographic activity in the lumbar paraspinal muscles of subjects with and without chronic low back pain. Physical Therapy, 65, $1347-1354$.

Morrison, D. P., \& Peck, D. F. (1990). Do self-report measures of affect agree? A longitudinal study. British Journal of Clinical Psychology, 29, 395-400.

Nigl, F. J., \& Fischer-Williams, M. (1980). Treatment of low back strain with electromyographic biofeedback and relaxation training. Psychosomatics, 21, 495-499.

Nouwen, A., \& Bush, C. (1984). The relationship between paraspinal EMG and chronic low back pain. Pain, 20, 109-123.

Rosnow, R. L., \& Rosenthal, R. (1989). Statistical procedures and the justification of knowledge in psychological science. American Psychologist, 44, 1276-1284.

Sherman, R. A. (1985). Relationships between strength of low back muscle contraction and reported intensity of chronic low back pain. American Joumal of Physical Medicine, 64, 190-200.

Sherman, R. A. (1988, November). Relationship between low back pain, stress, and continuous recordings of paraspinal surface EMG and movement in patients' normal environments. Paper presented at the joint annual meetings of the American Pain Society and the Canadian Pain Society, Toronto, Canada.

SPSS/PC+ Trends V2.0, [Computer Software]. (1987). Chicago, IL: SPSS Inc.

Turk, D. C., \& Rudy, T. E. (1986). Assessment of cognitive factors in chronic pain: A worthwhile enterprise? Journal of Consulting and Clinical Psychology, 54, 760-768. 
Wolf, S. L., \& Basmajian, J. V. (1978). Assessment of paraspinal electromyographic activity in normal subjects and in chronic back pain patients using a muscle biofeedback device. In E. Asmussen \& K. Jorgensen (Eds.), International series on biomechanics VB (pp. 319-324). Baltimore, MD: University Park Press.

Russe, T. C., \& Kutner, M. (1979). Normative data on low back mobility and activity levels. American Journal of Physical Medicine, 58, 217-229.

Wolf, S. L., Nacht, M., \& Kelly, J. L. (1982). EMG feedback training during dynamic movement for low back pain patients. Behavior Therapy, 13, 395-406. 